\title{
A Plane Quartic with Eight Undulations
}

\author{
By W. L. EdGE
}

(Received 11th August, 1948. Read 5th November 1948.)

1. The chief purpose of this paper is to demonstrate the existence of a plane quartic curve with eight undulations,' an "undulation" being a point at which the tangent has four-point contact. It is shown that the curve

$$
x^{4}-y^{4}-z^{4}+4 f x^{2} y z+2 f^{2} y^{2} z^{2}=0,
$$

where $(x, y, z)$ are homogeneous point-coordinates and $f$ a constant, has undulations at the eight points

$$
\begin{array}{llll}
(1,1,0) ; & (1,-1,0) ; & (1,0,1) ; & (1,0,-1) ; \\
(1, i, 0) ; & (1,-i, 0) ; & (1,0, i) ; & (1,0,-i) .
\end{array}
$$

The curve has, in addition to these undulations, eight inflections which are; in general, distinct. But there are two geometrically different possibilities of their not being distinct, and in either instance they coincide in pairs at four further undulations. Thus two types of curve arise without any ordinary inflections at all, their 24 inflections coinciding in pairs at 12 undulations.

The paper has been put together in consequence of encountering one of these two types of curve with twelve undulations that had not been previously recognised. A search through literature where this curve might have appeared led eventually to the paper "Sopra alcuhe curve del quarto ordine dotate di punti di ondulazione" by Masoni; 1 but the curve, notwithstanding the appearance of its equation during a digression, is passed over in silence. Yet the analysis on which Masoni embarked ought inevitably to have led to it; and the suspicion arises that when, because of some inadequacy or flaw in an investigation, a curve is overlooked something more may have escaped scrutiny as well. And once Masoni's mistake has been put right closer examination leads to the curve with eight undulations.

No elaborate technique is necessary for these modest researches, and none is used. The procedure followed is, in outline, to build up a figure in which there are two pencils $S$ and $S^{\prime}$ of quartic curves;

1 Rendiconto dell' accademia delle scienze fisiche e matematiche, 21 (Naples, 1882) $4 \tilde{5} \cdot 69$. 
the base points of either pencil consist of a set of four points reckoned four times over, each point of the set being an undulation on every curve of the pencil and the tangents at the four undulations being fixed lines. One set of four points figures as base points of $S$, a second set as base points of $S^{\prime}$. Moreover, the pencils are so related that there is a curve $q$ which belongs to $S$ and touches the curves of $S^{\prime}$ at their undulations while there is a curve $q^{\prime}$ which belongs to $S^{\prime}$ and touches the curves of $S$ at their undulations. It is then shown that $S$ and $S^{\prime}$ can be so chosen that they have a curve $\chi$ in common which will serve both as $q$ and as $q^{\prime}$; it is this curve $\chi$ which has eight undulations. A geometrical construction for such a curve is given in $\S 10$.

The enquiry into the possibility of there being further undulations is easy once the intersections of $\chi$ with its Hessian have been found; the work of $\S 13$ provides all the necessary information, and the two types of curve that possess twelve undulations are obtained in $\$ 14$. The explanation of the earlier encounter with one of these two types of curve is given in $\S 15$, and a few lines concerning the geometry of such a curve constitute $\S 16$. The last $\S 17$ points out the mistake Masoni made, and so explains why the curve eluded him.

2. Let $b$ and $c$ be two coplanar lines, $A$ their intersection. Suppose that a conic $\gamma$ meets $b$ in $P_{1}$ and $P_{2}$, its tangents at these two points being $t_{1}$ and $t_{2}$, while this same conic meets $c$ in $Q_{1}$ and $Q_{2}$, where its tangents are $u_{1}$ and $u_{2}$. When coordinates are introduced later the lines $b$ and $c$ will be taken for the sides $y=0$ and $z=0$ of the triangle of reference, and $x=0$ will be the polar $a$ of $A$ with respect to $\gamma$.

We shall use the pericil $S$ of quartic curves

$$
\Gamma^{2}+\lambda T_{1} T_{2} U_{1} U_{2}=0,
$$

where $\lambda$ is numerical and the capital letters give, when equated to zero, the equations of the curves denoted by the corresponding small letters. All the curves of $S$ have $\gamma$ for a contact conic, touching it at $P_{1} ; P_{2}$, $Q_{1}, Q_{2}$; and these four points are undulations on all the curves of $S$. The curves meet $b$ in $P_{1}$ and $P_{2}$, which are fixed, and in two further points which, as the curve varies in $S$, vary in an involution $I$. Similarly there arises an involution $J$ on $c$. Moreover the curves set up a $(1,1)$ correspondence $\Omega$ between the pairs of $I$ and the pairs of $J$, a pair of $I$ and a pair of $J$ corresponding to one another when they lie on the same quartic of $S$.

The involution $I$, it may be noted, includes the pair $P_{1}, P_{2}$; this is seen by choosing $\Gamma^{2}=0$ from $S$. If we choose $T_{1} T_{2} U_{1} U_{2}=0$ 
from $S$ it is seen that $I$ includes the pair $C_{1}, C_{2}$ of points where $b$ is met by $u_{1}$ and $u_{2}$. Since $I$ is determined by these two pairs it follows that $I$ is the involution cut on $b$ by those conics which touch $\gamma$ at $Q_{3}$ and $Q_{2}$. Similar considerations serve to show that $J$ is the involution cut on $c$ by those conics which touch $\gamma$ at $P_{1}$ and $P_{2} . \quad A$ is therefore a focus both of $I$ and of $J$. The other foci, $C$ of $I$ and $B$ of $J$, are the intersections of $b$ and $c$ with the polar $a$ of $A$ with respect to $\gamma$.

The curve of $S$ which passes through $A$ must, since it has to meet both $b$ and $c$ twice at $A$, have a node there; hence, in the correspondence $\Omega, A$ counted twice and reckoned as a pair of $I$ corresponds to $A$ counted twice and reckoned as a pair of $J$. This being so, there are sufficient data to determine $\Omega$ completely. For $\Omega$ is determined once it is known which pairs of $J$ correspond to any three pairs of $I$. The curve $\Gamma^{2}=0$ of $S$ shows that the pairs $P_{1}, P_{2}$ of $I$ and $Q_{1}, Q_{2}$ of $J$ correspond in $\Omega$; the curve $T_{1} T_{2} U_{1} U_{2}=0$ of $S$ shows that the pairs $C_{1}, C_{2}$ of $I$ and $B_{1}, B_{2}$ of $J$ correspond in $\Omega$, where $B_{1}$ and $B_{2}$ are the intersections of $c$ with $t_{1}$ and $t_{2}$.

3. There is a very simple way of setting up this correspondence $\Omega$ without any allusion to quartic curves. The diagonal points of the quadrangle $P_{1} P_{2} Q_{1} Q_{2}$ are $A$, the intersection of $P_{1} P_{2}$ and $Q_{1} Q_{2}$, $O$, the intersection of $P_{1} Q_{1}$ and $P_{2} Q_{2}$, and $O^{\prime}$, the intersection of $P_{1} Q_{2}$ and $P_{2} Q_{1}$. These same three points are, as is well known in the elementary geometry of conics, also the vertices of the diagonal triangle $\Delta$ of the quadrilateral $t_{1} t_{2} u_{1} u_{2}, O O^{\prime}$ being the line $a$ joining the intersection of $t_{1}$ and $t_{2}$ to the intersection of $u_{1}$ and $u_{2}$, and so on. $\Delta$ is self-polar for $\gamma$. Now the pairs of $I$ are joined to $O$ by the pairs of an involution $\omega$, of lines through $O$, whose focal rays are $O A$ and $O O^{\prime}$; and this same involution $\omega$, since it has the two requisite focal rays, has $J$ for its section by $c$. Suppose then that a pair of $I$ and a pair of $J$ are said to correspond to one another when they lie on the same pair of lines of $\omega$, that is, when they are in perspective from $O$. In this correspondence, as in $\Omega$, the pair $P_{1}, P_{2}$ of $I$ corresponds to the pair $Q_{1}, Q_{2}$ of $J$ and, also as in $\Omega$, to $A$ reckoned twice and regarded as a pair of $I$ corresponds $A$ reckoned twice and regarded as a pair of $J$. In order to establish the fact that this correspondence, set up by projection from $O$, between the pairs of $I$ and the pairs of $J$ is identical with $\Omega$ it is only necessary to show that the pair $B_{1}, B_{2}$ is projected from $O$ into $C_{1}, C_{2}$. And this follows rapidly on appealing to the cross-ratio property of $\gamma$, because

$$
B_{1} A Q_{1} Q_{2} \pi P_{1}\left(P_{1} P_{2} Q_{1} Q_{2}\right) \pi Q_{1}\left(P_{1} P_{2} Q_{1} Q_{2}\right) \pi P_{1} P_{2} C_{1} A \pi C_{1} A P_{1} P_{2}
$$


Thus the range $B_{1} A Q_{1} Q_{2}$ on $c$ is projective with the range $C_{1} A P_{1} P_{2}$ on $b$; the two ranges must therefore, since $A$ is common to them, be in perspective from the intersection of $P_{1} Q_{1}$ and $P_{2} Q_{2}$; and so $B_{1} C_{1}$ passes through $O$. As also, by an analogous argument, does $B_{2} C_{2}$.

The correspondence $\Omega$ between the pairs of $I$ and the paire of $J$ thus arises by projection from $O$. It is seen also, in the same fashion, to arise by projection from $O^{\prime}$, through which both $B_{1} C_{2}$ and $B_{2} C_{1}$ must pass.

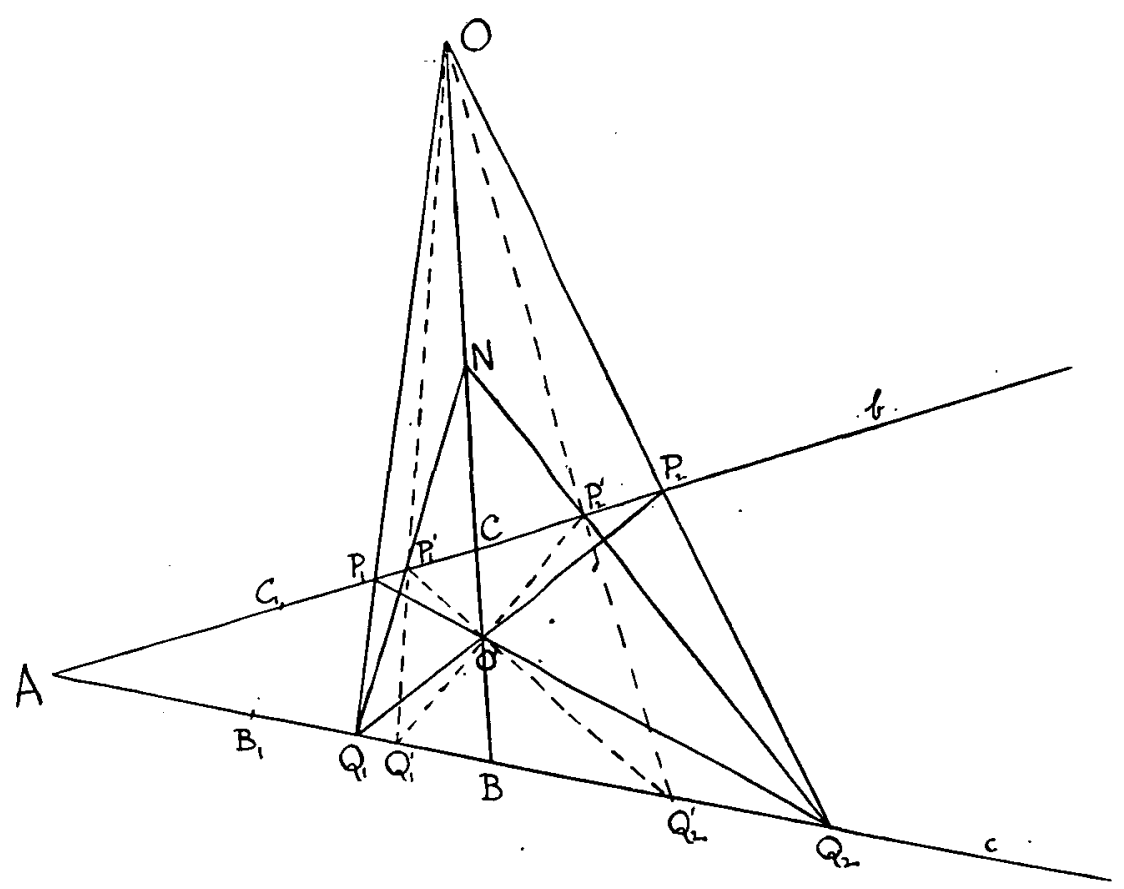

Fig. 1

4. Consider now a general curve $q$ of $S$. It meets $b$, apart from $P_{1}, P_{2}$, in a pair $P_{1}^{\prime}, P_{2}^{\prime}$. of $I$; it meets $c$, apart from $Q_{1}, Q_{2}$, in a pair $Q_{1}^{\prime}, Q_{2}{ }^{\prime}$ of $J$. Since the pairs $P_{1}^{\prime}, P_{2}^{\prime}$ and $Q_{1}{ }^{\prime}, Q_{2}{ }^{\prime}$ lie on the same curve $q$ they correspond in $\Omega$; hence we may suppose that $P_{1}{ }^{\prime} Q_{1}{ }^{\prime}$ and $P_{2}{ }^{\prime} Q_{2}{ }^{\prime}$ meet in $O$ while $P_{1}{ }^{\prime} Q_{2}{ }^{\prime}$ and $P_{2}{ }^{\prime} Q_{1}{ }^{\prime}$ meet in $O^{\prime}$. Moreover: since $\gamma$ is a contact conic of $q$, and since the pair of lines $b$ and $c$ pass through the four contacts of $\gamma$ and $q$ and meet $q$ further in $P_{1}{ }^{\prime}, P_{2}{ }^{\prime}, Q_{1}{ }^{\prime}, Q_{2}{ }^{\prime}$ there is a contact conic $\gamma^{\prime}$ touching $q$ at these last four points. And $\gamma^{\prime}$ also has $\Delta$ for a self-polar triangle. It should however be noted that $P_{1}{ }^{\prime}, P_{2}{ }^{\prime}, Q_{1}{ }^{\prime}, Q_{2}{ }^{\prime}$ need not be, and in general will not be, undulations on $q$. 


\section{a Plane Quartic with Eight Undolations}

5. The remarks so far made may be illustrated by a metrical example. Let $A$ be the origin of rectangular coordinates $X$ and $Y, b$ and $c$ the bisectors of the angles between the axes, $\gamma$ the circle $X^{2}+Y^{2}=2$; then $O U^{\prime} B C$, the polar of $A$ with respect to $\gamma$, is the "line at infinity." Since $P_{1}$ and $P_{2}$, a pair of $I$, are equidistant from its focus $A I$ consists of those pairs of points on $b$ whose joins are bisected at $A$. Similarly for $J$ on c. Perspectivity from $O$ is by means of parallels to the $X$-axis, perspectivity from $O^{\prime}$ by means of parallels to the $Y$-axis. If $P_{1}^{\prime}, P_{2}^{\prime}$ is any pair of $I$, and $Q_{1}^{\prime}, Q_{2}^{\prime}$ the corresponding pair of $J, P_{1}^{\prime} P_{2}{ }^{\prime} Q_{1}^{\prime} Q_{2}^{\prime}$ is a square whose sides are parallel to the axes and whose centre is at $A$. The pencil $S$ is

$$
\left(X^{2}+Y^{2}-2\right)^{2}+\lambda(2+X+Y)(2-X+Y)(2+X-Y)(2-X-Y)=0 ;
$$

every curve of $S$ has.four axes of symmetry, namely the two coordinate axes and the lines $b$ and $c$, and its contact conic $\gamma^{\prime}$ is the circle circumscribing the square $P_{1}^{\prime} P_{2}^{\prime} Q_{1}^{\prime} Q_{2}^{\prime}$.

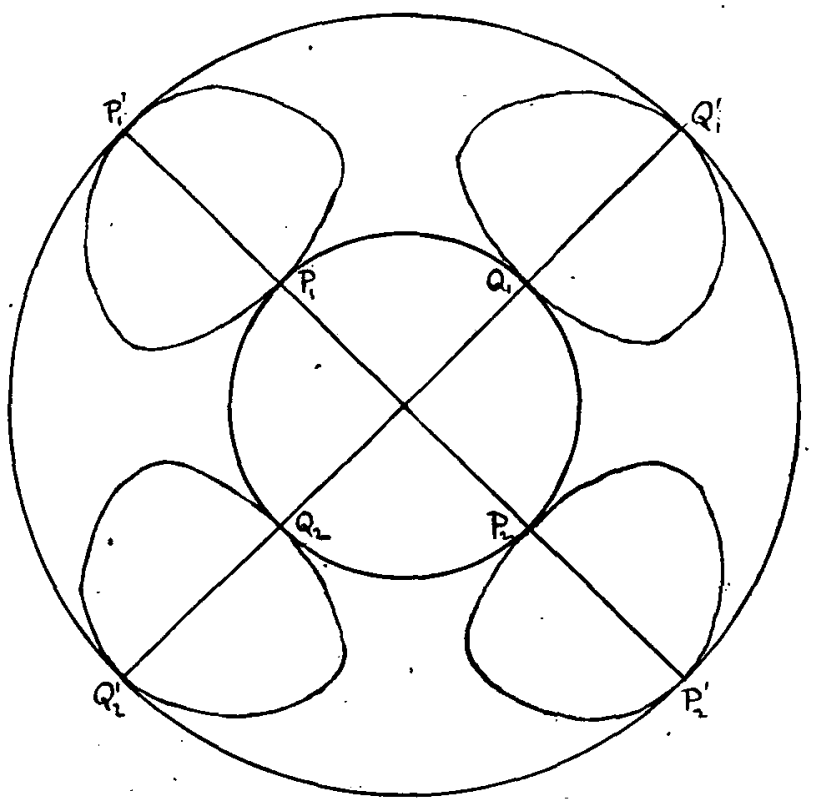

Fig. 2

If we take $\lambda=1$ there arises the curve $q$ whose equation

$$
\left(X^{2}+Y^{2}-2\right)^{2}+(2+X+Y)(2-X+Y)(2+X-Y)(2-X-Y)=0
$$

may also be written

$$
\left(X^{2}+Y^{2}-2\right)\left(X^{2}+Y^{2}-10\right)+\left(X^{2}-Y^{2}\right)^{2}=0,
$$

a form of equation which not only makes manifest the two concentric quadritangent circles $\gamma$ and $\gamma^{\prime}$ but also shows that $q$ lies entirely in the annulus between them. The points of contact with $\gamma^{\prime}$ are not undulations ; for, since $q$ is confined to the annulus, its curvature at each contact with $\gamma^{\prime}$ must exceed the curvature of $\gamma^{\prime}$ whereas at an undu- 
6. We have constructed the curve $q$ with contact conics which, if it is desired to exhibit their points of contact, may be denoted by $\gamma\left(P_{1} P_{2} Q_{1} Q_{2}\right)$ and $\gamma^{\prime}\left(P_{1}^{\prime} P_{2}^{\prime} Q_{1}^{\prime} Q_{2}^{\prime}\right)$. We now show that $q$ has also contact conics $\vartheta\left(P_{1} P_{2} Q_{1}^{\prime} Q_{2}^{\prime}\right)$ and $\vartheta^{\prime}\left(P_{1}^{\prime} P_{2}^{\prime} Q_{1} Q_{2}\right)$. For we may write, if $y=0$ and $z=0$ are the equations of $b$ and $c$,

$$
\Gamma \equiv \rho y^{2}-T_{1} T_{2} \equiv \sigma z^{2}-U_{1} U_{2}
$$

where $\rho$ and $\sigma$ are numerical, whereupon the identity

$$
\begin{aligned}
&\left\{\rho y^{2}-(1+\lambda) T_{1} T_{2}\right\}\left\{\sigma z^{2}-(1+\lambda) U_{1} U_{2}\right\}+\lambda \rho \sigma y^{2} z^{2} \\
& \equiv(1+\lambda)\left\{\left(\rho y^{2}-T_{1} T_{2}\right)\left(\sigma z^{2}-U_{1} U_{2}\right)+\lambda T_{1} T_{2} U_{1} U_{2}\right\}
\end{aligned}
$$

instantly discloses the new contact conics. But the proviso must be made that $\lambda$ is not -1 , or that $q$ must not be the curve of $S$ which passes through $A$. It may be noted in passing that $\vartheta$ may be defined as the conic which touches $\gamma$ at $P_{1}$ and $P_{2}$ and passes through either $Q_{1}^{\prime}$ or $Q_{2}^{\prime}$, or else as the conic which touches $\gamma^{\prime}$ at $Q_{1}^{\prime}$ and $Q_{2}^{\prime}$ and passes through either $P_{1}$ or $P_{2}$. There are similar definitions for $\vartheta^{\prime}$.

For the special curve $q$ of $\S 5$ the existence of $\vartheta$ and $\vartheta^{\prime}$ is visually clear from the figure; $\vartheta$ is the ellipse whose axes are $P_{1} P_{2}$ and $Q_{1}^{\prime} Q_{2}^{\prime}$ while $\vartheta^{\prime}$ is the ellipse whose axes are $P_{1}^{\prime} P_{2}^{\prime}$ and $Q_{1} Q_{2}$. The relation of these ellipses to the curve is displayed by writing its equation in the form

$$
\left(3 X^{2}+4 X Y+3 Y^{2}-10\right)\left(3 X^{2}-4 X Y+3 Y^{2}-10\right)+\left(X^{2}-Y^{2}\right)^{2}=0 .
$$

7. We now amplify the figure by introducing the second pencil $S^{\prime}$ of quartic curves

$$
\Gamma^{\prime 2}+\lambda^{\prime} T_{1}^{\prime} T_{2}^{\prime} U_{1}^{\prime} U_{2}^{\prime}=0
$$

where $t_{1}^{\prime}, t_{2}^{\prime}, u_{1}^{\prime}, u_{2}^{\prime}$ are the tangents of $\gamma^{\prime}$ at $P_{1}^{\prime}, P_{2}^{\prime}, Q_{1}^{\prime}, Q_{2}^{\prime}$ respectively. All these curves have undulations at $P_{1}^{\prime}, P_{2}^{\prime}, Q_{1}^{\prime}, Q_{2}{ }^{\prime}$ with $\gamma^{\prime}$ as contact conic. They cut an involution on $b$ and this, being the same as the involution given by the conics which touch $\gamma^{\prime}$ at $Q_{1}{ }^{\prime}$ and $Q_{2}{ }^{\prime}$, is $I$; similarly the quartics cut $J$ on $c$. Moreover they set up a $(1,1)$ correspondence between the pairs of $I$ and the pairs of $J$ and this, being the same $(1,1)$ correspondence as is determined by the perspectivities whose centres are $O$, the intersection of $P_{1}^{\prime} Q_{1}^{\prime}$ and $P_{2}^{\prime} Q_{2}^{\prime}$, and $O^{\prime}$, the intersection of $P_{1}{ }^{\prime} Q_{2}^{\prime}$ and $P_{2}^{\prime} Q_{1}^{\prime}$, is identical with the correspondence $\Omega$ which was set up by quartica of the pencil $S$. Hence the curve $q^{\prime}$ of $S^{\prime}$ which passes through $P_{1}$ passes also through $P_{2}$, which is paired with $P_{1}$ in $I$, and through $Q_{1}$ and $Q_{2}$, which constitute the pair of $J$ which corresponds in $\Omega$ to the pair $P_{1}$ and $P_{2}$ of $I$. Thus there is a curve $q^{\prime}$ with undulations at $P_{1}^{\prime}, P_{2}{ }^{\prime}, Q_{1}{ }^{\prime}, Q_{2}{ }^{\prime}$ and passing through $P_{1}, P_{2}, Q_{1}, Q_{2}$. These latter points are contacts. 
of $q^{\prime}$ with a conic which, we can prove, is $\gamma$. For it will, as meeting $\gamma$ in $P_{1}, P_{2}, Q_{1}, Q_{2}$, coincide with $\gamma$ if it touches $\gamma$ at any one of these four points, say at $P_{1}$. Now it will touch $\gamma$ at $P_{1}$ if it touches $\vartheta$ at $P_{1}$, and it will touch $\vartheta$ at $P_{1}$ if $q^{\prime}$ touches $\vartheta$ at $P_{1}$. But, since there are conics which touch $q^{\prime}$ at both sets of four points $P_{1}^{\prime} P_{2}^{\prime} Q_{1}^{\prime} Q_{2}^{\prime}$ and $P_{1} P_{2} Q_{1} Q_{2}$ so, as has been seen in $\S 6$ for $q$, must there be conics which touch $q^{\prime}$ at both sets of four points $P_{1}^{\prime} P_{2}^{\prime} Q_{1} Q_{2}$ and $P_{1} P_{2} Q_{1}{ }^{\prime} Q_{2}{ }^{\prime}$; and the last of these conics, as touching $\gamma^{\prime}$ at $Q_{1}^{\prime}$ and $Q_{2}^{\prime}$ and passing through $P_{1}$, can only be $\vartheta$.

As for the curve $q^{\prime}$ which arises in Fig. 2, its equation is

$$
5\left(X^{2}+Y^{2}-2\right)\left(X^{2}+Y^{2}-10\right)=\left(X^{2}-Y^{2}\right)^{2} \text {. }
$$

It consists of two ovals, one outside $\gamma^{\prime}$ and having undulations at its four contacts with $\gamma^{\prime}$, the other inside $\gamma$.

8. We have now built up a figure with the following properties:

(i) Quartic curves of a pencil $S$ have undulations at $P_{1}, P_{2}, Q_{1}, Q_{2}$ with $\gamma$ as contact conic; one curve $q$ of $S$ passes through $P_{1}^{\prime}, P_{2}^{\prime}, Q_{1}^{\prime}, Q_{2}^{\prime}$ with $\gamma^{\prime}$ as contact conic.

(ii) Quartic curves of a pencil $S^{\prime}$ have undulations at $P_{1}^{\prime}, P_{2}{ }^{\prime}, Q_{1}{ }^{\prime}, Q_{2}{ }^{\prime}$ with $\gamma^{\prime}$ as contact conic; one curve $q^{\prime}$ of $S^{\prime}$ passes through $P_{1}, P_{2}, Q_{1}, Q_{2}$ with $\gamma$ as contact conic.

These curves $q$ and $q^{\prime}$ therefore touch at eight points, four of which are undulations on $q$ and the other four undulations on $q^{\prime}$.

It may be remarked that if, in building up the figure, we choose for $q$ the degenerate curve of $S$ made up of the four lines $t_{2}, t_{2}, u_{1}, u_{2}$, then the points $P_{1}{ }^{\prime}, P_{2}{ }^{\prime}, Q_{1}{ }^{\prime}, Q_{2}{ }^{\prime}$ coincide, for this particular choice of $q$, with $C_{1}, C_{2}, B_{1}, B_{2}$ respectively. There is a conic $\gamma^{\prime}$ touching, at these four points, $u_{1}, u_{2}, t_{1}, t_{2}$, which therefore coincide, for this particular choice of $q$, with $t_{1}^{\prime}, t_{2}^{\prime}, u_{1}^{\prime}, u_{2}^{\prime}$ respectively. Thus the same degenerate quartic belongs both to $S$ and to $S^{\prime}$. We proceed now to investigate the possibility of the same non-degenerate quartic belonging both to $S$ and to $S^{\prime}$; for if such a curve can be found it will have eight undulations. The question then is whether the curve $q$ of (i) and the curve $q^{\prime}$ of (ii) can coincide.

This is a crucial question, for if it can be answered in the affirmative we shall have obtained a curve with eight undulations. Such a curve would have, in addition to $\gamma$ and $\gamma^{\prime}$, also $\vartheta$ and $\vartheta^{\prime}$ touching it at four undulations and its equation would, for appropriate values of the 
constants $\lambda, \lambda^{\prime}, \mu, \mu^{\prime}, k, l, m, n$ have to admit all the following forms:

$$
\begin{array}{ll}
\Gamma^{2}+\lambda T_{1} T_{2} U_{1} U_{2}=0, & \Theta^{2}+\mu T_{1} T_{1} U_{1}^{\prime} U_{2}^{\prime}=0, \\
\Gamma^{\prime 2}+\lambda^{\prime} T_{1}^{\prime} T_{2}{ }^{\prime} U_{1}^{\prime} U_{2}^{\prime}=0, & \Theta^{\prime 2}+\mu^{\prime} T_{1}^{\prime} T_{2}^{\prime} U_{1} U_{2}=0, \\
\Gamma \Gamma^{\prime}+k y^{2} z^{2}=0, & \Theta \Theta^{\prime}+l y^{2} z^{2}=0, \\
m y^{4}+T_{1} T_{2} T_{1}^{\prime} T_{2}^{\prime}=0, & n z^{4}+U_{2} U_{2} U_{1}^{\prime} U_{2}^{\prime}=0
\end{array}
$$

the last two forms being associated with the two sets of four collinear undulations on $b$ and $c$ respectively. All these.forms will actually be exhibited in $\$ 11$, but we first proceed to examine what geometrical restriction is imposed on the figure in order that it should conform to this new requirement.

9. Let it then be postulated that $q$ and $q^{\prime}$ are the same curve $q$, with eight undulations. The curves which touch $\vartheta^{\prime}$ at $P_{1}^{\prime}, P_{2}^{\prime}, Q_{1}, Q_{2}$ and have these points as undulations constitute the pencil

$$
\Theta^{\prime 2}+\mu^{\prime} T_{1}^{\prime} T_{2}^{\prime} U_{1} U_{2}=0 \text {. }
$$

The involution which these curves cut on $b$ is the same as that cut by the conics which touch $\vartheta^{\prime}$ at $Q_{1}$ and $Q_{2}$ and so is $I$; similarly the curves cut $J$ on $c$. But the $(1,1)$ correspondence which the curves set up between the pairs of $I$ and the pairs of $J$ is not $\Omega$, which had centres of perspectivity at $O$ and $O^{\prime}$, but a new correspondence with a centre of perspectivity at $N$, the intersection of $P_{1}^{\prime} Q_{1}$ and $P_{2}^{\prime} Q_{2}$. This point $N$ lies on the polar of $A$ with respect to $\vartheta^{\prime}$, i.e. on $O O^{\prime}$ [see Fig. 1. In Fig. $2 P_{1}^{\prime} Q_{1}$ and $P_{2}^{\prime} Q_{2}$ are parallel]. Now $q$, which touches $\vartheta^{\prime}$ at each of $P_{1}^{\prime}, P_{2}^{\prime}, Q_{1}, Q_{2}$ has, according to our present hypothesis; undulations at these four points and so belongs to the pencil now under consideration; hence the pair $P_{1}, P_{2}$ of $I$ in which $q$ meets $b$ has to be in perspective from $N$ with the pair $Q_{2}{ }^{\prime}, Q_{2}{ }^{\prime}$ of $J$ in which $q$ meets $c$ and so $N P_{1}$ must pass either through $Q_{1}^{\prime}$ or through $Q_{2}^{\prime}$. But, by the harmonic property of the quadrangle $P_{1} P_{1}{ }^{\prime} Q_{1}{ }^{\prime} Q_{1}$, the lines $P_{1} Q_{1}{ }^{\prime}$ and $P_{1}^{\prime} Q_{1}$ intersect on $A O^{\prime}$, so that $N P_{1}$ cannot pass through $Q_{1}^{\prime}$ unless $N$ coincides with $O^{\prime}$. We must therefore presume that $N P_{1}$ passes through $Q_{2}{ }^{\prime}$ [this does not happen in the figures] and so $N Q_{1}{ }^{\prime}$ through $P_{2}$. Hence, by successive projections from $N$ and $O$,

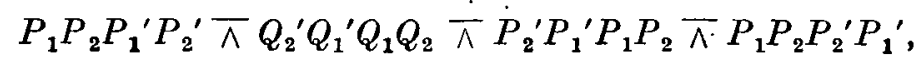

showing that $P_{1}^{\prime}, P_{2}^{\prime}$ must be harmonic to $P_{1}, P_{2}$, This, then, is a necessary condition for $q$ and $q^{\prime}$ to coincide. It involves of course also that $Q_{1}^{\prime}, Q_{2}^{\prime}$ must be harmonic to $Q_{1}, Q_{2}$. 
When the focus $A$ and the pair $P_{1}, P_{2}$ of $I$ are given there is one and only one pair $P_{1}^{\prime}, P_{2}^{\prime}$ of $I$ that is harmonic to $P_{1}, P_{2}$; the harmonic relation can therefore be fulfilled. We now show that its fulfilment is sufficient to ensure that $q$ has eight undulations.

Suppose that the geometry is as depicted in Fig. 1, but with the additional restriction of the harmonic relation being fulfilled. Then

$$
A Q_{1} Q_{2}{ }^{\prime} B \bar{\wedge} A P_{1} P_{2}{ }^{\prime} C \bar{\wedge} C P_{1} P_{1}{ }^{\prime} A \sqcap A P_{1}{ }^{\prime} P_{1} C,
$$

the points of the third set being respectively the harmonic conjugates of the points of the second set in regard to $P_{1}, P_{2}$. This projective relation shows that $P_{1} Q_{2}^{\prime}$ passes through the intersection of $P_{1}{ }^{\prime} Q_{1}$ and $C B$, that is through $N$. Similar reasoning shows that $P_{2} Q_{1}^{\prime}$ also passes through $N$.

Let $\gamma$ be any (non-degenerate) conic through $P_{1}, P_{2}, Q_{1}, Q_{2}$. We can construct, in virtue of the perspectivity centred at $O$, a curve $q$ with undulations at $P_{1}, P_{2}, Q_{1}, Q_{2}$ and a curve $q^{\prime}$ with undulations at $P_{1}{ }^{\prime \prime}, P_{2}{ }^{\prime \prime}, Q_{1}{ }^{\prime}, Q_{2}{ }^{\prime} ; q$ and $q^{\prime}$ touch at all eight points and have the four contact conics.

$\gamma\left(P_{2} P_{2} Q_{1} Q_{2}\right), \quad \gamma^{\prime}\left(P_{1}{ }^{\prime} P_{2}{ }^{\prime} Q_{1}{ }^{\prime} Q_{2}{ }^{\prime}\right), \quad \vartheta\left(P_{1} P_{2} Q_{1}{ }^{\prime} Q_{2}{ }^{\prime}\right), \quad \vartheta^{\prime}\left(P_{1}{ }^{\prime} P_{2}{ }^{\prime} Q_{1} Q_{2}\right)$.

But now, in virtue of the perspectivity centred at $N$, we can construct a curve $r$ having undulations at $P_{1}, P_{2}, Q_{1}, Q_{2}^{\prime}$ with $\vartheta$ as contact conic and passing through $P_{1}{ }^{\prime}, P_{2}{ }^{\prime}, Q_{1}, Q_{2} ; r$ has (cf. \&6) $\gamma, \gamma^{\prime}$ as contact conics, and therefore also $\vartheta^{\prime}$. Similarly we can construct a curve $r^{\prime}$, having the same four contact conics, with undulations at $P_{1}{ }^{\prime} ; P_{2}{ }^{\prime}, Q_{1}, Q_{2}$ and passing through $P_{1}, P_{2}, Q_{1}{ }^{\prime}, Q_{2}{ }^{\prime}$. There thus apparently arise, four quartic curves $q, q^{\prime}, r, r^{\prime}$ all touching one another at the same eight points. But if two plane quartics touch one another at eight distinct points they can only have two-point intersection at each of them; should they, at any one of the eight points, have more than two-point intersection they cannot be distinct curves. If however two curves have a common undulation with the same tangent they have four-point intersection there. It follows that $q$ must coincide both with $r$ and with $r^{\prime}$, and these with $q^{\prime}$; there is only a single curve $\chi$ and it has undulations at all the eight points.

10. In order to obtain a plane quartic with eight undulations we could therefore proceed as follows.

Take involutions $I$ and $J$ one on each of any two coplanar lines; both $I$ and $J$ are to have the intersection of the lines for 
a focus. Take any pair $P_{1}, P_{2}$ of $I$ and any pair $Q_{1}, Q_{2}$ of $J$; there is a unique pair $P_{1}^{\prime}, P_{2}^{\prime}$ of $I$ harmonic to $P_{1}, P_{2}$ and a unique pair $Q_{1}^{\prime}, Q_{2}^{\prime}$ of $J$ harmonic to $Q_{1}, Q_{2}$. Associated with each non-singular conic $\gamma$ through $P_{1}, P_{2}, Q_{1}, Q_{2}$ there is a quartic curve having undulations at all the eight points and $\gamma$ for a contact conic.

This construction enables us quickly to calculate the freedom of quartics with eight undulations. For two constants are required to fix each line, one to fix each focus (other than the intersection) of $I$ and $J$, one to select any pair of $I$, one to select any pair of $J$; so that at this stage eight constants have been used. Any conic through $P_{1}, P_{2}, Q_{1}, Q_{2}$ is then chosen to play the part of $\gamma$, and as the choice involves the assignation of one constant the freedom of the quartic curve is 9 . Alternatively: we may choose the two lines and $\gamma$ all at random, thus using $2+2+5=9$ constants. The figure is then completely determined since both $I$ and $J$ are fixed when $\gamma$ and the lines are given.

11. We now introduce homogeneous coordinates for which $A B C$ is the triangle of reference while the unit point is at the concurrence of $A O, B P_{1}, C Q_{2}$; that these three lines do actually concur follows from the fact that $P_{1} Q_{2}$ passes through the harmonic conjugate $O^{\prime}$ of $O$ with respect to $B$ and $C$. Then the coordinates of the points are $P_{1}:(1,0,1) ; \quad P_{2}:(1,0,-1) ; \quad Q_{1}:(1,-1,0) ; \quad Q_{2}:(1,1,0)$; and we may suppose that

$$
\Gamma \equiv x^{2}-y^{2}-z^{2}+2 f y z
$$

where $f$ is a constant whose square is not equal to 1 . We then take, in order to satisfy the harmonic relation, $P_{1}^{\prime}:(i, 0,1) ; \quad P_{2}^{\prime}:(-i, 0,1) ; \quad Q_{1}^{\prime}:(-i, 1,0) ; \quad Q_{2}^{\prime}:(i, 1,0)$ where $i^{2}=-1$, and it is then found that

$$
\begin{aligned}
\Theta & \equiv x^{2}+y^{2}-z^{2}+2 f y z, \\
\Theta^{\prime} & \equiv x^{2}-y^{2}+z^{2}+2 f y z, \\
\Gamma^{\prime} & \equiv x^{2}+y^{2}+z^{2}+2 f y z .
\end{aligned}
$$

Since we wish all four conics $\gamma, \gamma^{\prime}, \vartheta, \vartheta^{\prime}$ to be non-singular the fourth power of $f$ must not be equal to 1. Further: on polarising the above quadratic forms we obtain 
$T_{1} \equiv x+f y-z, \quad T_{2} \equiv x-f y+z, \quad U_{1} \equiv x+y-f z, \quad U_{2} \equiv i \quad \quad y+f z$, $T_{1}{ }^{\prime} \equiv i x+f y+z, \quad T_{2}^{\prime} \equiv-i x+f y+z, \quad U_{1}^{\prime} \equiv-i x+y+f z, \quad U_{2}^{\prime} \equiv i x+y+f z$, so that

$$
\begin{aligned}
& \left(f^{2}+1\right) \Gamma^{2}-2 T_{1} T_{2} U_{1} U_{2} \equiv\left(f^{2}-1\right) \Sigma \equiv\left(f^{2}+1\right) \Gamma^{\prime 2}-2 T_{1}{ }^{\prime} T_{2}{ }^{\prime} U_{1}{ }^{\prime} U_{2}{ }^{\prime}, \\
& \left(f^{2}-1\right) \Theta^{2}+2 T_{1} T_{2} U_{1}{ }^{\prime} U_{2}^{\prime} \equiv\left(f^{2}+1\right) \Sigma \equiv\left(f^{2}-1\right) \Theta^{\prime 2}+2 T_{1}{ }^{\prime} T_{2}{ }^{\prime} U_{1} U_{2},
\end{aligned}
$$

where

$$
\begin{aligned}
\Sigma & \equiv x^{4}-y^{4}-z^{4}+4 f x^{2} y z+2 f^{2} y^{2} z^{2} \\
& \equiv \Gamma \Gamma^{\prime}+2\left(f^{2}-1\right) y^{2} z^{2} \equiv \Theta \Theta^{\prime}+2\left(f^{2}+1\right) y^{2} z^{2} \\
& \equiv\left(f^{4}-1\right) y^{4}+T_{1} T_{2} T_{1}{ }^{\prime} T_{2}{ }^{\prime} \equiv\left(f^{4}-1\right) z^{4}+U_{1} U_{2} U_{1}{ }^{\prime} U_{2}{ }^{\prime} .
\end{aligned}
$$

The curve $\chi$ whose equation is $\Sigma=0$ thus has eight undulations. It would degenerate into four lines, and so have an infinity of undulations, if $f^{4}=1$; but this particularisation has already been excluded.

12. The line coordinates of the tangents of $\chi$ at its undulations are

$$
\begin{array}{llll}
(1, f,-1) ; & (1,-f, 1) ; & (1,1,-f) ; & (1,-1, f) ; \\
(i, f, 1) ; & (-i, f, 1) ; & (-i, 1, f) ; & (i, 1, f) ;
\end{array}
$$

it is clear that all these eight lines belong to the envelope $m n+f l^{2}=0$.

Now every inflectional tangent of a quartic curve belon "s to the harmonic envelope, and if the tangent is not merely inflection $/$ but undulatory it is a double line of the harmonic envelope; thus the eight undulatory tangents of $\chi$ are all double lines of its harmonic envelope. But this envelope is of class 6 and so, having eight tangents of a conic as double lines, must include every tangent of the conic. Thus the harmonic envelope of $\chi$ consists of the conic $m n+f l^{2}=0$ together with an envelope of class 4 to which also belong the tangents at the eight undulations of $\chi$.

13. A non-singular plane quartic possesses twenty-four inflections; they constitute the set of intersections of the quartic with its Hessian and are, in general, all distinct. But if the quartic has an undulation this counts for two inflections and, as Cayley remarked, the Hessian touches the quartic there. Thus the Hessian $h$ of $\chi$ touches $\chi$ at each of the eight undulations. There remain eight further points common to $h$ and $\chi$; these are, in general, ordinary inflections as we shall show. 
Of the twenty-four intersections of $\chi$ with the sextic curve $h$ the sixteen which consist of the eight contacts taken twice make up the complete intersection of $\chi$ and the quartic $y^{2} z^{2}=0$. The remaining eight must therefore make up the complete intersection of $\chi$ with some conic $\epsilon$. And there must be an identity

$$
H \equiv \Sigma E^{\prime}+y^{2} z^{2} E
$$

where $H$ is some numerical multiple of the Hessian determinant of $\Sigma, E=0$ the equation of $\epsilon, E^{\prime}$ another quadratic form.

Some features of this identity can be discerned before its complete form is worked out in detail. Since no odd power of $x$ appears in $\Sigma, \chi$ is invariant for the harmonic inversion $W$ whose fundamental spaces are $A$ and $a$. Moreover: since $h$ is a covariant curve of $\chi$ it, too, must be invariant for $W$, so that no odd power of $x$ can appear in $H$. The cemplete set of intersections of $\dot{h}$ and $\chi$ is invariant for $W$; but so, as we already know, is the set of eight undulations of $\chi$. Thus the set of eight intersections of $\chi$ and $\epsilon$ is invariant for $W$, so that no odd power of $x$ can occur in $E$. Thus every symbol, apart from $E^{\prime}$, in 13.1 represents a polynomial with no odd power of $x$ occurring in it, so that $E^{\prime}$ also represents such a polynomial. Next: since

$$
\frac{\partial^{2} \Sigma}{\partial y^{2}} \equiv 4\left(f^{2} z^{2}-3 y^{2}\right) . \quad \text { and } \quad \frac{\hat{o}^{2} \Sigma}{\partial z^{2}} \equiv 4\left(f^{2} y^{2}-3 z^{2}\right)
$$

the polar conics of both $B$ and $C$ with respect to $\chi$ are line-pairs, so that $B$ and $C$ lie on $h$. Thus $H$ is without the terms in $y^{6}$ and $z^{6}$, and so $E^{\prime}$ must be without the terms in $y^{2}$ and $z^{2}$. Thus only the two terms in $x^{2}$ and $y z$ can appear in $E^{\prime}$. We now take

$$
19 \dot{2} H \equiv\left|\begin{array}{ccc}
12 x^{2}+8 f y z & 8 f x y & 8 f x z \\
8 f x y & 4 f^{2} z^{2}-12 y^{2} & 4 f x^{2}+8 f^{2} y z \\
8 f x z & 4 f x^{2}+8 f^{2} y z & 4 f^{2} y^{2}-12 z^{2}
\end{array}\right|
$$

and find the coefficients $\alpha, \eta, \alpha^{\prime}, \beta^{\prime}, \eta^{\prime}$ so that

$$
H \equiv\left(\alpha x^{2}+2 \eta y z\right) \Sigma+\left(\alpha^{\prime} x^{2}+\beta^{\prime} y^{2}+\beta^{\prime} z^{2}+\dot{2}_{\eta^{\prime}} y z\right) y^{2} z^{2},
$$

the coefficients of $y^{2}$ and $z^{2}$ being presumed equal in $E$ because of the symmetry in $y$ and $z$. We find that actually

$$
H \equiv f^{2}\left(2 f y z-x^{2}\right) \Sigma+3\left(1-f^{4}\right)\left(2 f y z+3 x^{2}\right) y^{2} z^{2} .
$$

The equation of $\epsilon$ is therefore $2 f y z+3 x^{2}=0$. 
14. Consider the intersections of $\chi$ and $\epsilon$; they are inflections of $\chi$ and, as can now be seen, are in general distinct. Both $\chi$ and $\epsilon$ are invariant for $W$, so that each of their intersections which does not lie on $a$ is paired with a second one, the join of the pair passing through $A$. But $a$, whose equation is $x=0$, either meets $\epsilon$ in $B$ and $C$, neither of which lies on $\chi$, or else, if $f=0$, belongs entirely to $\epsilon$, in which event the common points of $\chi$ and $\epsilon$ coincide in pairs at four points of $a$ and $\chi$ becomes the curve $x^{4}=y^{4}+z^{4}$ with four undulations on each side of $\Delta$-twelve undulations in all. The only other way in which any of the eight intersections can coalesce is by the four lines through $A$ which join them in pairs not being distinct. Now it appears, on eliminating $x$ between the equations of $\chi$ and $\epsilon$, that these four lines are given by

$$
9 y^{4}+2 f^{2} y^{2} z^{2}+9 z^{4}=0
$$

and so are distinct except when $f^{4}=81$; when $f$ is a fourth root of 81 the four lines coalesce two by two into a repeated line-pair and $\epsilon$ becomes a contact conic of $\chi$. The eight inflections of $\chi$ thus coincide in pairs and there again arises a quartic with twelve undulations.

To summarise: if $f^{4}$ is not equal to 1 the curve $\Sigma=0$ has, in general, eight undulations and eight inflections. Exceptions only occur when $f^{4}$ is either 0 or 81 , and the special quartics corresponding to such values of $f$ have twelve undulations.

15. The two types of plane quartic with twelve undulations are indeed both already known; but whereas the one, having $f=0$, is the curve first encountered by Dyck in his researches ${ }^{1}$ on regular Riemann surfaces the other, having $f^{4}=81$, has only recently, been noticed. Since $\Sigma$ is unchanged when $f, x, y$ are simultaneously replaced by if, $i x$, iy the four fourth roots of 81 give curves that are projectively equivalent; suppose then that $f=3$, when the curve is

$$
x^{4}-y^{4}-z^{4}+12 x^{2} y z+18 y^{2} z^{2}=0 .
$$

If $y$ is replaced by $\frac{1}{2}(Y+Z)$ and $z$ by $\frac{1}{2}(Y-Z)$, the triangle of reference thus being changed from $A B C$ to $A O O^{\prime}$, the equation of the curve becomes

$$
x^{4}+Y^{4}+Z^{4}=3\left(Y^{2} Z^{2}+Z^{2} x^{2}-x^{2} Y^{2}\right)
$$

and this, when $i Z$ is written for $Z$, is precisely the form given recently

1 Hath. Annalen, 17 (1880), 512. 
[Edinburgh Math. Notes, No.35 (1945), p. 11] for a certain curve with twelve undulations. This Note was elementary: there was no intention to do more than give the equation of the curve and establish the existence of the undulations. But it may not be out of place to declare here that the curve was found in the course of investigating the quartic surfaces that have been called Maschke surfaces, and so might itself be called a Maschke curve. It arose thus.

A Maschke surface $M$ possesses ten azygetic tetrahedra. ${ }^{1}$ The first polar, with respect to $M$, of any vertex $V$ of any one of these tetrahedra $U$ consists of the opposite face $v$ of $U$ together with a quadric $\Psi$. Both $M$ and $\Psi$ are unchanged by harmonic inversion in $V$ and $v$, as is therefore also their common curve of order eight; any point of this curve (other than its eight intersections with $v$ ) is thus joined to $V$ by a line which meets the curve a second time. Thus a singly-infinite set of lines, bitangents of $M$, pass through $V$ and generate a quartic cone $\mu$. The Maschke curve arises as a plane section of $\mu$, and that it must possess twelve undulations can be deduced from the geometry of the Maschke surface.

Analytically: we may take the equation of $M$ to be

$$
x^{4}+y^{4}+z^{4}+t^{4}=6\left(y^{2} z^{2}+z^{2} x^{2}+x^{2} y^{2}+x^{2} t^{2}+y^{2} t^{2}+z^{2} t^{2}\right),
$$

and the tetrahedron of reference is one of the azygetic tetrahedra. The first polar of its vertex $x=y=z=0$ consists of the opposite face $t=0$ together with the quadric

$$
t^{2}=3\left(x^{2}+y^{2}+z^{2}\right) \text {. }
$$

The equation of the cone $\mu$ is the result of eliminating $t$ between the equations of this quadric and of $M$, and so is

$$
\begin{gathered}
x^{4}+y^{4}+z^{4}+9\left(x^{2}+y^{2}+z^{2}\right)^{2}=6\left(y^{2} z^{2}+z^{2} x^{2}+x^{2} y^{2}\right)+18\left(x^{2}+y^{2}+z^{2}\right)^{2}, \\
x^{4}+y^{4}+z^{4}+3\left(y^{2} z^{2}+z^{2} x^{2}+x^{2} y^{2}\right)=0 .
\end{gathered}
$$

This equation actually appears in Masoni's paper; it is difficult not to regard this as a somewhat fortuitous apparition and it is curious that, in a paper devoted to the study of quartic curves with undulations, a curve that actually has twelve should appear without there being the slightest sign of suspicion of its possessing any at all.

16. The geometry of the Dyck curve is well known; only a few lines need be written about the Maschke curve by way of relating its properties to those mentioned in the Note.

I Proc. Edinburgh Math. Soc. (2), 7 (1945), 154. 
When $f=3$ the equation of the contact conic $\epsilon$ is $2 y z+x^{2}=0$ and its contacts are its intersections with the lines $y^{2}+z^{2}=0$; thus the four further undulations also form a quadrangle with $A, O, O^{\prime}$ as its diagonal points, and their actual coordinates are

$$
(1-i, 1, i) ; \quad(i-1,1, i) ; \quad(1+i, 1,-i) ; \quad(-i-1,1,-i) .
$$

Two of their six joins meet at $A$; any one of the four other joins meets $b$ and $c$ each in one of the original eight undulations. Thus, as was pointed out in the Note, the twelve undulations lie four on each of six lines and make up with $A, O, O^{\prime}$ the complete set of intersections of these lines. The tangents of the curve at those of its undulations which lie on a pair of lines whose intersection is one of $A, O, O^{\prime}$ are eight tangents of the same conic, and the three conics so arising constitute the harmonic envelope of the Maschke curve. Each undulatory tangent touches two of the three conics. When the curve is given by 15.1 its harmonic envelope is

$$
\left(3 l^{2}+m^{2}+n^{2}\right)\left(l^{2}+3 m^{2}+n^{2}\right)\left(l^{2}+m^{2}+3 n^{2}\right)=0 .
$$

The freedom of either a Dyck curve or a Maschke curve can be found from the geometry of the curves themselves; but since we know that they are both obtainable from a curve of freedom 9 by fixing the value of a constant otherwise arbitrary it follows that they both have 'freedom 8.

17. Quartics which have undulations have scarcely been studied save in the one paper by Masoni. When Masoni wrote the Dyck. curve was a recent discovery and his paper culminates with some account of it; but the curve with eight undulations, with its particularised Maschke form, eluded him. The reason why this happened is that, on p. 66 of his paper, Masoni jumped to the conclusion that when two equations represent the same curve their left-hand sides are identical; but in fact either may be any constant non-zero multiple of the other.

It is a straightforward matter to transcribe into our notation what Masoni in fact does; he takes for triangle of reference that formed by the lines $t_{1}, t_{2}, b$ and considers the quartic in relation to the contact conics that we have called $\gamma$ and $\vartheta$. We have already given the relevant identities, namely

and

$$
2 T_{1} T_{2} U_{1} U_{2}-\left(1+f^{2}\right) \Gamma^{2} \equiv\left(1-f^{2}\right) \Sigma,
$$

$$
2 T_{1} T_{2} U_{1}^{\prime} U_{2}^{\prime}-\left(1-f^{2}\right) \Theta^{2} \equiv\left(1+f^{2}\right) \Sigma \text {. }
$$


These may be written, $\lambda, \lambda^{\prime}$ being numerical constants,

$$
\begin{aligned}
& \lambda T_{1} T_{2} U_{1} U_{2}-\left\{T_{1} T_{2}-\left(1-f^{2}\right) y^{2}\right\}^{2} \equiv \frac{1-f^{2}}{1+f^{2}} \Sigma \\
& \lambda^{\prime} T_{1} T_{2} U_{1}^{\prime} U_{2}^{\prime}-\left\{T_{1} T_{2}+\left(1+f^{2}\right) y^{2}\right\}^{2} \equiv \frac{1+f^{2}}{1-f^{2}} \Sigma .
\end{aligned}
$$

The left-hand sides of these two identities cannot therefore be identically equal unless

$$
\begin{gathered}
\frac{1-f^{2}}{1+f^{2}}=\frac{1+f^{2}}{1-f^{2}}, \\
f=0,
\end{gathered}
$$

when the quartic is a Dyck curve. It is because of this that Masoni, who assumes that the two left-hand sides are identically equal, obtains only the Dyck curve and makes it, by his false assumption, inevitable that the curve with eight undulations will elude him. The mistake seems to have remained unnoticed ever since it was made in 1882 .

Mathematical Institute, UNIVERSITY OF EDINBURGH. 